\title{
Dificultades de estudiantes universitarios en la comprensión de textos argumentativos*
}

\author{
Guadalupe Alvarez ${ }^{1}$ \\ (iD http://orcid.org/0000-0001-7152-730X \\ Mónica Beatriz García ${ }^{2}$ \\ (D) $h t t p: / / o r c i d . o r g / 0000-0002-2739-3161$ \\ Universidad Nacional de General Sarmiento, Argentina
}

DOI: http://dx.doi.org/10.17081/eduhum.19.32.2529

Recibido: 26 de junio de 2016

Aceptado: 25 de septiembre 2016

\begin{abstract}
Students' difficulties to comprehend argumentative texts
Palabras clave:

Universidad,

Comprensión textual,

Argumentación, Moodle.

\section{Resumen}

Este artículo se encuadra en un proyecto mayor que se lleva a cabo en el Taller de Lectoescritura de la Universidad Nacional de General Sarmiento (Buenos Aires, Argentina) con el fin de mejorar las habilidades de lectura y escritura de los estudiantes a partir de materiales digitales. Aquí se presenta una de las experiencias realizada en 2012 y 2013: se trata de una serie de actividades en la plataforma educativa Moodle de la universidad como forma de repaso para el segundo parcial, centrado en textos argumentativos. El objetivo es analizar las dificultades que han enfrentado los estudiantes al completar dos cuestionarios orientados al reconocimiento de los elementos básicos de textos argumentativos. Esto ha permitido concluir que, para comprender este tipo de textos, es necesario el reconocimiento de sus aspectos fundamentales (cuestión polémica, tesis y argumentos) y, para ello, se deben establecer guías pedagógicas en consonancia con abordajes teóricos específicos sobre el texto argumentativo y su comprensión.
\end{abstract}

Key words:

University,

Textual comprehension, Argumentation, Moodle.

\begin{abstract}
This paper focuses on a project related to a workshop in literacy conducted by the Universidad Nacional de General Sarmiento (Buenos Aires, Argentina) in order to improve one's reading and writing skills through digital resources. This paper shows one of the experiences conducted in 2012 and 2013 as follow: A series of activities related to argumentative texts are designed as a review for second partial exam, in the institutional Moodle platform. The purpose of this research is to analyze the students' difficulties to complete two questionnaires which aim to recognize the basic elements of the argumentative texts. In conclusion, in order to comprehend this type of text, it is necessary to recognize the main aspects (controversial issue, thesis and arguments) and it is relevant to establish some pedagogical guidelines according to the specific theoretical approaches related to argumentative texts and their comprehension.
\end{abstract}

Referencia de este artículo (APA): Álvarez, G. \& García, M. (2017). Dificultades de estudiantes universitarios en la comprensión de textos argumentativos. En Revista Educación y Humanismo, 19(32), 18-30. http://dx.doi.org/10.17081/eduhum.19.32.2529

* Este artículo es derivado del proyecto "Las prácticas de enseñanza de la escritura mediadas por tecnologías digitales. Estudio de casos en los niveles medio y superior en Argentina" (2015-2017), financiado por la Universidad Nacional General Sarmiento y el Consejo de Investigaciones Científicas y Técnicas, dirigido por Guadalupe Álvarez y codirigido por Laura Ferrari en la Universidad Nacional General Sarmiento. Se agradece a esta institución por la financiación de dicho proyecto y al Consejo de Investigaciones Científicas y Técnicas por el soporte a la primera autora.

1. Doctora en Letras. Profesora y Licenciada en Letras. Investigadora del Consejo Nacional de Investigaciones Científicas y Técnicas y Docente investigadora del Instituto del Desarrollo Humano de la Universidad Nacional General Sarmiento, Argentina. galvarez@ungs.edu.ar

2. Especialista en Ciencias Sociales con mención en Lectura, Escritura y Educación, Licenciada en Lengua y Literatura. Docente investigadora en el Instituto del Desarrollo Humano de la Universidad Nacional General Sarmiento, Argentina.

mobegar@gmail.com 


\section{Introducción}

Las dificultades que los estudiantes universitarios enfrentan para resolver las tareas típicas de lectura y escritura en la universidad, tanto al ingresar como a lo largo de las carreras, ha sido el tema central de una serie importante de investigaciones (Ameijde, Murga, Padilla \& Douglas, 2000, 2002; Estienne \& Carlino, 2004; Carlino, 2004, 2005; García \& Álvarez, 2010; Lacon de De Lucia \& Ortega de Hocevar, 2004; Narvaja de Arnoux, Di Stefano \& Pereira, 2002; Piacente \& Tittarelli, 2006; Riestra, 1999; Solé \& Castells, 2004; Vázquez \& Miras, 2004).

Ameijde et al. (2002) afirma en concreto que, entre los obstáculos que enfrentan los estudiantes, se destacan la comprensión y la producción de discursos argumentativos. Frente a este panorama, algunos investigadores (Di Stéfano \& Pereira, 2002; Pérez \& Vega, 2002; Dib, 2007; Sanjuán, 2009; Nothstein \& Valente, 2010) se han preguntado sobre las posibles intervenciones didácticas que mejorarían la comprensión de textos argumentativos.

Pérez y Vega (2002) consideran que, para alcanzar una competencia argumentativa y desarrollar un pensamiento crítico, no basta argumentar intuitivamente, sino que es necesario conocer el uso, por una parte, de algunas reglas fundamentales para elaborar argumentos de diversa índole y, por otra, de determinadas estrategias discursivas que potencian su eficacia.

Por su parte, Di Stéfano y Pereira (2002) aseveran que el abordaje de los textos argumentativos debe permitir la reflexión sobre las regulaciones impuestas por la situación de interacción discursiva, sus relaciones con el interdiscurso, y sus condiciones de producción y de circulación. Por ello, proponen una reflexión sobre aspectos de la argumentación que consideran especialmente relevantes para el desarrollo de habilidades de lectura y escritura académicas en los estudiantes que ingresan a la universidad, a saber: el análisis de las características de la secuencia argumentativa, su dimensión enunciativa, los géneros en que suele plasmarse, la ejecución y descripción de planes textuales propios de la argumentación, los recursos más frecuentemente empleados para plasmar los argumentos y el modo de construcción de los razonamientos (Arnoux, Di Stéfano \& Pereira, 2002, p.49).

Paralelamente, Nothstein y Valente (2011) consideran que la interpretación de los textos argumentativos implica una fuerte interacción entre texto y lector porque no solo cuentan las representaciones del lector, sino también las habilidades de lectura que demanda el texto. Las autoras consideran necesario, entonces, explicitar las habilidades puestas en juego en la lectura de un texto argumentativo en las actividades de comprensión que diseñe el docente. Este tipo de reflexión le permitiría al estudiante ser consciente de sus propios procesos cognitivos y también le brindaría al docente la posibilidad de mejorar los dispositivos didácticos, a los efectos de un mejor desempeño de aquellos. Para lograr estas metas, Nothstein y Valente indagan 
las posibilidades del modelo dialogal que plantea Plantin $(1998,2005)$ por considerar que este brinda claves relevantes para la enseñanza de la lectura de discursos argumentativos. Según Plantin (1998), una determinada situación de lenguaje empieza a ser argumentativa cuando se manifiesta en ella una oposición discursiva, que se plantea a partir de un problema en forma de pregunta o cuestión polémica. Es decir, en la argumentación, se presenta la confrontación de un discurso y de un contra-discurso, orientados por una misma cuestión polémica.

Para las ya citadas Nothstein y Valente (2011), el diseño de actividades pedagógicas de acuerdo con el modelo de Plantin $(2005,1998)$ permite al lector prestar atención a las estrategias argumentativas de cada autor y resemantizarlas al leerlas como instancias de un discurso en el que se pueden leer las huellas del contradiscurso respecto de una cuestión polémica. Así, el modelo permite contemplar la dimensión contextual, asociando las prácticas discursivas a ámbitos específicos de la vida social.

En definitiva, las reflexiones de los autores anteriores permitirían sugerir que, como explica Solana (2002), los conocimientos metalingüísticos, es decir, los conocimientos que los estudiantes tengan sobre los textos argumentativos como objeto, inciden notablemente sobre sus procesos comprensivos.

En el presente trabajo, se ha asumido, como indica también Solana (2002), que para promover la actividad metalingüística, que permite la adquisición de conocimientos metalingüísticos relativos a lo argumentativo, es necesario que la guía pedagógica que proponga el docente esté diseñada de acuerdo con una teoría o teorías de la argumentación bien delimitadas. En este caso, siguiendo a Nothstein y Valente (2011), se han diseñado y puesto a prueba actividades elaboradas a partir del modelo dialogal de Plantin (1998, 2005). En específico, se ha propiciado el reconocimiento de los elementos fundamentales del texto argumentativo, a saber: cuestión polémica, postura (o hipótesis) del autor del texto frente a esa cuestión y argumentos esgrimidos para avalar la hipótesis.

Por otra parte, el estudio expuesto en este artículo se encuadra en un proyecto de investigación mayor que se lleva a cabo en el marco del Taller de Lectoescritura (TLE) del Curso de Aprestamiento Universitario (CAU) de la Universidad Nacional de General Sarmiento (UNGS) (Buenos Aires, Argentina), con el objeto de mejorar las habilidades de lectura y escritura de los estudiantes, a partir de materiales especialmente diseñados para entornos virtuales de aprendizaje. Este artículo se centra en una de las experiencias, realizada en 2012 y 2013: una serie de actividades diseñadas con los cuestionarios Moodle. Acorde con ello, el objetivo del presente trabajo es analizar las dificultades de los estudiantes en dos cuestionarios orientados al reconocimiento de los elementos básicos de textos argumentativos, lo que, por otra parte, permite extraer conclusiones sobre futuros abordajes de la comprensión de ese tipo de textos. 


\section{Método}

Desde el punto de vista metodológico, el proyecto mayor en el que se encuadra el presente estudio asume el diseño de una investigaciónacción que construye el conocimiento por medio de la práctica (Hernández, Fernández \& Baptista, 2010). Se trata de un diseño que parte de problemas concretos y situados, y busca intervenir las problemáticas diagnosticadas para transformarlas. En esta investigación, entonces, los investigadores responsables, junto con docentes involucrados en la experiencia, trabajan de manera sucesiva con estudiantes del TLE. Esta es una de las materias del Curso de Aprestamiento Universitario (CAU), que debe ser aprobado para ingresar a UNGS. Dicha materia, que se cursa de manera presencial dos veces por semana, hace especial hincapié en la comprensión y producción de textos explicativos y argumentativos. Las instancias de evaluación del TLE abarcan, fundamentalmente, dos parciales presenciales y un trabajo final. El primer parcial está dedicado a la lectura y escritura de textos explicativos. El segundo parcial, a la lectura y el resumen de textos argumentativos. El trabajo final consiste en la elaboración de un informe de lectura o una monografía.

Como toda investigación-acción, este trabajo comprende diversas actividades cuya ejecución se puede representar como una "espiral sucesiva de ciclos" (Hernández et al., 2010). Las actividades son las siguientes: clarificación, profundización y diagnóstico de los problemas de escritura específicos del grupo de estudiantes; formulación de las secuencias didácticas en Moodle; implementación de las secuencias y su evaluación teniendo en cuenta la percepción de los estudiantes y la evolución de su escritura; y, por último, retroalimentación, la cual conduce a un nuevo diagnóstico y a una nueva espiral de reflexión y acción.

Como diagnóstico sobre las habilidades de lectura y escritura del grupo de estudiantes del TLE, se han tomado en cuenta informes e investigaciones realizadas durante 2009-2011 (Álvarez, García \& Qués, 2010; García \& Álvarez, 2010). Teniendo en cuenta los resultados de esas investigaciones, se han diseñado actividades a partir de herramientas de Moodle como estrategias de repaso para los parciales y los trabajos prácticos fundamentales.

Es importante destacar que hemos utilizado la plataforma Moodle de la UNGS que, como toda plataforma educativa, cuenta con un conjunto de instrumentos capaces de gestionar todos los aspectos estructurales de un espacio virtual de formación. Así, Moodle ofrece la posibilidad de utilizar diferentes recursos y herramientas con fines educativos, entre los cuales se destacan los foros, los chats, las wikis, los cuestionarios (con la inclusión de preguntas de diferente tipo) y las encuestas, así como la posibilidad de compartir archivos y sitios de Internet.

Este artículo, se centra en el "Repaso para el segundo parcial", repaso que comprende un espacio virtual en Moodle compuesto por dos textos argumentativos y tres cuestionarios para 
abordar su comprensión, así como la producción de sus resúmenes. Como se ha adelantado, la selección de textos y la elaboración de los cuestionarios se realizó en función del enfoque de Plantin (1998, 2005), a partir del cual también se han elaborado las actividades de las clases presenciales.

El texto 1, titulado "La revolución comunicacional", del filósofo y periodista José Pablo Feinmann, es una nota de opinión publicada en el diario Tiempo Argentino. El texto 2, titulado "El agua, ¿de quién es?”, del poeta y periodista Juan Gelman, es también una nota de opinión, publicada en el diario Página/12.

A partir de la lectura de cada texto, se ha propuesto a los estudiantes la resolución de tres cuestionarios: un cuestionario orientado a la comprensión del texto argumentativo, otro orientado a la identificación de los elementos del texto argumentativo, y un tercer cuestionario dirigido a la identificación de errores en el resumen del texto argumentativo. Todos los cuestionarios son de tipo "opción múltiple" y de autocorrección. Así, las respuestas, en general, ofrecen tres alternativas. Por otra parte, una vez que el estudiante completa el cuestionario y lo envía, el sistema le indica si su respuesta es correcta o no y por qué.

En el presente artículo, se analiza el desempeño de los estudiantes en dos cuestionarios sobre identificación de los elementos del texto argumentativo: el Cuestionario 1, que incluye consignas relativas al texto 1 (ver Anexo 1) y el Cuestionario 2, referido al texto 2 (ver Anexo 2).
Ambos cuestionarios se han puesto a prueba en ocho comisiones de la materia a lo largo de dos años (2012-2013), con un total de 250 estudiantes. Sin embargo, no todos los estudiantes ingresaron y completaron las actividades, en parte porque estas no se presentaron como obligatorias. Así, 114 estudiantes resolvieron el Cuestionario 1 y 64, el Cuestionario 2.

Para analizar el desempeño de los estudiantes en estos cuestionarios, se han cuantificado, por cada pregunta, la cantidad y el porcentaje de las tres alternativas de respuestas. Esto ha permitido reconocer no solo el porcentaje de las respuestas correctas, sino también la respuesta incorrecta más seleccionada. Es importante destacar que no se trata de un estudio estadístico, por lo que las cantidades y los porcentajes solo ilustran algunas tendencias. De todos modos, a partir de estas tendencias, se han extraído conclusiones relativas al tipo de dificultades que enfrentan los estudiantes en la lectura de textos argumentativos, lo cual permitirá ajustar los materiales digitales para próximas implementaciones.

\section{Resultados}

El primer cuestionario fue resuelto por 103 estudiantes, cuyo desempeño se muestra en la Tabla 1. En esta tabla se resalta la cantidad y el porcentaje relativos a la respuesta adecuada:

Como se puede observar en la Tabla 1, las preguntas 1, 2, 3, 4 y 6 están asociadas con los porcentajes más altos de respuestas adecuadas (78,64\%; 78,64\%; 80,59\%, 77,66\% y 81,55\%, respectivamente). La pregunta 5 , por el contrario, ha presentado mayor dificultad para 
Tabla 1. Desempeño de los estudiantes en cada pregunta del Cuestionario 1 analizado

\begin{tabular}{|c|c|c|c|c|c|c|}
\hline RESPUESTAS & 1 & 2 & 3 & 4 & 5 & 6 \\
\hline A & $\begin{array}{c}6 \\
5,82 \%\end{array}$ & $\begin{array}{c}2 \\
1,94 \%\end{array}$ & $\begin{array}{c}14 \\
13,59 \%\end{array}$ & $1413,59 \%$ & $65,82 \%$ & $8481,55 \%$ \\
\hline B & $\begin{array}{c}15 \\
14,56 \%\end{array}$ & $\begin{array}{c}19 \\
18,44 \%\end{array}$ & $\begin{array}{c}6 \\
5,82 \%\end{array}$ & $\begin{array}{c}80 \\
77,66 \%\end{array}$ & $\begin{array}{c}42 \\
40,73 \%\end{array}$ & $1312,63 \%$ \\
\hline $\mathrm{C}$ & $\begin{array}{c}81 \\
78,64 \%\end{array}$ & $\begin{array}{c}81 \\
78,64 \%\end{array}$ & $\begin{array}{c}83 \\
80,59 \%\end{array}$ & $\begin{array}{c}8 \\
7,77 \%\end{array}$ & $5553,45 \%$ & $65,82 \%$ \\
\hline No contesta esa pregunta & $\begin{array}{c}1 \\
0,98 \%\end{array}$ & $\begin{array}{c}1 \\
0,98 \%\end{array}$ & - & $\begin{array}{c}1 \\
0,98 \%\end{array}$ & - & - \\
\hline TOTAL DE PREGUNTAS & 103 & 103 & 103 & 103 & 103 & 103 \\
\hline
\end{tabular}

Fuente: Elaboración propia

Tabla 2. Desempeño de los estudiantes en cada pregunta del Cuestionario 2 analizado

\begin{tabular}{|c|c|c|c|c|c|c|}
\hline RESPUESTAS & 1 & 2 & 3 & 4 & 5 & 6 \\
\hline A & $\begin{array}{c}10 \\
17,21 \%\end{array}$ & $\begin{array}{c}3 \\
5,17 \%\end{array}$ & $\begin{array}{c}19 \\
32,75 \%\end{array}$ & $3356,89 \%$ & $813,79 \%$ & $813,79 \%$ \\
\hline B & $\begin{array}{c}7 \\
12,06 \% \\
\end{array}$ & $\begin{array}{c}3 \\
5,17 \% \\
\end{array}$ & $\begin{array}{c}4 \\
6,89 \% \\
\end{array}$ & $\begin{array}{c}9 \\
15,51 \% \\
\end{array}$ & $\begin{array}{c}40 \\
68,96 \%\end{array}$ & $2746,55 \%$ \\
\hline $\mathrm{C}$ & $\begin{array}{c}41 \\
70,68 \% \\
\end{array}$ & $\begin{array}{c}52 \\
89,65 \% \\
\end{array}$ & $\begin{array}{c}34 \\
58,62 \% \\
\end{array}$ & $\begin{array}{c}16 \\
27,58 \% \\
\end{array}$ & $1017,24 \%$ & $2339,65 \%$ \\
\hline No contesta esta pregunta & - & - & $\begin{array}{c}1 \\
1,17 \% \\
\end{array}$ & - & - & - \\
\hline TOTAL DE PREGUNTAS & 58 & 58 & 58 & 58 & 58 & 58 \\
\hline
\end{tabular}

Fuente: Elaboración propia

los estudiantes: solo un poco más del 40 \% logró responderla adecuadamente.

El segundo cuestionario fue resuelto por 58 estudiantes. El desempeño de estos se muestra en la Tabla 2, en la cual también aparecen resaltados la cantidad y el porcentaje relativos a la respuesta adecuada:

En la Tabla 2 se aprecia que las preguntas 1, 2 y 5 están asociadas con los porcentajes más altos de respuestas correctas $(70,68 \%, 89,65 \%$ y $68,96 \%$, respectivamente). En cuanto a las preguntas 3 y 4, un poco más del $50 \%$ logró responder adecuadamente (58,62\% y 56,89\%, respectivamente), mientras que menos del $50 \%$ respondió bien la $6(46,55 \%)$.

Los resultados observados en las Tablas 1 y 2 sugieren que los estudiantes reconocen $\sin$ mayores dificultades la cuestión polémica y la hipótesis del texto, al menos cuando estos elementos se encuentran en posiciones iniciales y de manera explícita. También reconocen la inclusión de citas de autoridad como una de las estrategias del texto argumentativo.

Por el contrario, los estudiantes tienen 
problemas para establecer adecuadamente los argumentos. En particular, seleccionan respuestas que son incompletas como argumentos o que dan cuenta de aspectos secundarios de las ideas expresadas por el autor. Por otra parte, tal como indican los resultados obtenidos en la respuesta 6 de la Tabla 2, los estudiantes realizarían lecturas literales de algunas frases sin considerar las relaciones con frases previas del texto, y el modo en que se correlacionan para expresar sentido.

En síntesis, en relación con ambos textos, la mayor dificultad de los estudiantes está representada por el reconocimiento adecuado de los argumentos, sobre todo en el texto 2. En este sentido, cabe destacar que mientras en el texto 1 una de las preguntas sobre los argumentos se asocia con un desempeño bajo; en el texto 2 , las tres preguntas relativas a los argumentos muestran porcentajes bajos de respuestas adecuadas.

\section{Conclusiones}

En este artículo, se han presentado los resultados de un estudio relativo de las experiencias concretadas en 2012 y 2013 en el TLE de la UNGS: una serie de cuestionarios que se han diseñado en Moodle como forma de repaso para el segundo parcial, centrado en textos argumentativos. En particular, se han analizado las dificultades de los estudiantes en dos cuestionarios orientados al reconocimiento de los elementos básicos de dos textos argumentativos. Esto ha permitido extraer conclusiones sobre futuros abordajes de la comprensión de ese tipo de textos.
En principio, lo analizado parece indicar que, como plantean otros autores (Arnoux et al., 2002; Pérez Rifo \& Vega Alvarado, 2002), para lograr la comprensión de textos argumentativos, es necesario que los estudiantes reconozcan aspectos fundamentales de este tipo de textos, a saber: la cuestión polémica, la hipótesis, los argumentos y las estrategias más frecuentemente empleados para plasmar los argumentos.

Por otra parte, como plantea Solana (2002), la guía pedagógica para alcanzar este reconocimiento debería estar en consonancia con abordajes teóricos específicos. En el estudio llevado a cabo, el modelo dialogal de Plantin (2005) ha brindado un marco conceptual muy fructífero para propiciar en los estudiantes la lectura adecuada de discursos argumentativos. Así, es posible sugerir que el reconocimiento de la cuestión polémica contribuye con la identificación de la hipótesis del texto, fundamentalmente en aquellos casos en que su enunciación es explícita o fácilmente deducible.

Sin embargo, el abordaje propuesto aún sería insuficiente como ayuda para el reconocimiento de los argumentos, lo que estaría evidenciado en las dificultades de los estudiantes para resolver las preguntas vinculadas con dichos elementos del texto argumentativo.

En este sentido, sería necesario ajustar las ayudas pedagógicas a fin de lograr que los estudiantes reconozcan los argumentos de manera completa, y no en función de ideas parciales o secundarias. Por un lado, sería oportuno entrenar 
de manera más metódica y profunda el modo de construcción de los razonamientos (Arnoux et al., 2002). Y por otro, se requiere trabajar la lectura de textos argumentativos en función de lecturas no meramente superficiales, en las cuales se pongan en juego estrategias inferenciales para la comprensión. Una posibilidad podría ser apuntar al diseño de ejercicios que incluyan un específico entrenamiento en la autoexplicación, lo que ya se ha puesto a prueba con textos explicativos (Álvarez, Bassa \& Ferrari, 2013).

El desafío pendiente, entonces, es seguir investigando para ofrecer guías pedagógicas cada vez más eficaces que contribuyan a mejorar la comprensión lectora de nuestros estudiantes. La tecnología puede contribuir con este desafío siempre que se esté integrada a la propuesta didáctica diseñada.

\section{Referencias}

Álvarez, G., Bassa, L. \& Ferrari, L. (noviembre de 2013). Estrategias para favorecer la comprensión de textos explicativos en el ingreso a la universidad: hacia una propuesta con integración de tecnologías digitales. Ponencia presentada en el VII Congreso Internacional Cátedra Unesco para el Mejoramiento de la Calidad y Equidad de la Educación en América Latina, con base en la Lectura y la Escritura, Córdoba, Argentina.

Álvarez, G., García, M. \& Qués, M.E. (2010). Entornos virtuales de aprendizaje y didáctica de la lengua. Una propuesta para mejorar las habilidades de reformulación productiva de estudiantes preuniversitarios. Revista Q, 5(9), 1-24.

Ameijde, D., Murga, M., Padilla, C. \& Douglas, S. (2002). Representaciones de las dificultades discursivas en estudiantes universitarios. Revista del INSIL, 15, 11-32.

Ameijde, D., Murga, M., Padilla, C. \& Douglas, S. (agosto de 2000). Conceptualizaciones sobre el saber lingüístico en el discurso estudiantil. Ponencia presentada en el Congreso Nacional de Lingüística, Sociedad Argentina de Lingüística, Mar del Plata, Argentina.

Arnoux, E., Di Stéfano, M. \& Pereira, C. (2002). La argumentación. La lectura y la escritura en la universidad. Buenos Aires: Eudeba.

Carlino, P. (2004). El proceso de escritura académica: cuatro dificultades de la enseñanza universitaria. Educere: Revista Venezolana de Educación, 8(26), 321-327. Carlino, P. (2005). Escribir, leer y aprender en la universidad. Una introducción a la alfabetización académica. Buenos Aires: Fondo de Cultura Económica.

Dib，J. (2007). ¿Cómo leer la dimensión argumentativa de los textos académicos? En I. Klein et al., El taller del escritor universitario (pp.62-73). Buenos Aires: Prometeo.

Di Stéfano, M. \& Pereira, C. (julio de 2002). La enseñanza de la argumentación en el nivel superior. Propuestas y experiencias de trabajo en los niveles de grado y de 
posgrado. Ponencia presentada en el Congreso Internacional "La argumentación”, Buenos Aires, Argentina.

Estienne V. \& Carlino P. (junio de 2004). Leer en la universidad. Enseñar y aprender una cultura nueva. Ponencia presentada en el $7^{\circ}$ Congreso Internacional de promoción de la Lectura y el Libro, Buenos Aires, Argentina.

Feinmann, P. La revolución comunicacional. Tiempo Argentino. Recuperado de http:// aballay-calidejacobacci.blogspot.com. ar/2011/08/la-revolucion-comunicacional-por-jose.html

Gelman, J. El agua, ¿de quién es? Página/12. Recuperado de http:// www.pagina 12 .com.ar/diario/ contratapa/13-171938-2011-07-10.html

García, M. \& Álvarez, G. (2010). Hacia una propuesta superadora de las dificultades de alumnos preuniversitarios en reformulaciones productivas del texto fuente. Revista Onomazein, 21(1), 191-223.

Hernández Sampieri, R., Fernández Collado, C. \& Baptista Lucio, P. (2010). Metodología de la investigación ( $5^{\mathrm{a}} \mathrm{ed}$.). México: McGraw-Hill.

Lacon de De Lucia, N. \& Ortega de Hocevar, S. (julio de 2004). La problemática de la escritura en la universidad: una propuesta de solución a partir de la articulación con el Polimodal. Ponencia presentada en el I Congreso Internacional Educación, Lenguaje y Sociedad: "Tensiones Educativas en América Latina”, Santa Rosa, Argentina.
Narvaja de Arnoux, E., Di Stefano, M. \& Pereira, C. (2002). La lectura y la escritura en la universidad. Buenos Aires: EUDEBA.

Pérez Rifo, M. \& Vega Alvarado, O. (2002). Enseñanza del discurso argumentativo: la argumentación por valores. Onomazein, 7 , 403-411.

Piacente, T. \& Tittarelli, A. (2006). Comprensión y producción de textos en alumnos universitarios: la reformulación textual. Orientación y Sociedad, 6, 99-126.

Plantin, C. (1998). La argumentación. Barcelona: Editorial Ariel.

Plantin, C. (2005). L'argumentation. Histoire, théories et perspectives. París: PUF.

Riestra, D. (1999). Reenseñar la escritura a estudiantes universitarios. Revista Infancia y Aprendizaje, 22(88), 43-56.

Sanjuán Varela, O. (2009). La metacognición como estrategia pedagógica en la producción de textos argumentativos. Revista Educación y Humanismo, 17, 40-52.

Solana, Z. (julio de 2002). Comprensión de textos escritos académicos y enseñanza de la argumentación. Ponencia presentada en el Congreso Internacional "La argumentación". Buenos Aires, Argentina.

Solé, I. \& Castells, N. (2004). Aprender mediante la lectura y la escritura: ¿Existen diferencias en función del dominio disciplinar? Lectura y Vida, 25(4), 6-17.

Vázquez, A. \& Miras, M. (2004). Cómo se representan los estudiantes universitarios en las tareas de escritura. Ponencia presentada en la Reunión Mente y Cultura: Cambios 
Representacionales en el Aprendizaje, Centro Regional Universitario Bariloche de la Universidad Nacional del Comahue, Bariloche, Argentina.

\section{Anexo 1. Cuestionario 1}

\section{Pregunta 1}

En la primera oración del texto, Feinmann explicita cuál es el TEMA del texto. Sin embargo, la temática señalada en esa oración es muy general. Si tuvieras que especificar un poco más el tema, ¿cuál sería la opción adecuada?

\section{Seleccione una respuesta.}

a. El texto habla de la historia de los medios de comunicación.

b. El texto habla de los medios de comunicación y la importancia de la técnica para su desarrollo.

c. El texto habla del poder de los medios en la actualidad.

\section{Pregunta 2}

Leer con atención el siguiente fragmento: “quien hundió al proletariado -aún no del todo pero en eso todavía está muy empeñada- fue la burguesía capitalista. Pero la revolución no consiste tanto en haber derrotado al comunismo sino en alzar triunfalista el poder de los medios de comunicación".

Luego, determinar qué frase de las siguientes sintetiza más adecuadamente las ideas expresadas por el autor en la oración subrayada.
Seleccione una respuesta.

a. El autor considera que la verdadera revolución de la burguesía capitalista ha sido vencer al comunismo imponiendo el capitalismo.

b. El autor cuestiona que la verdadera revolución de la burguesía ha sido controlar los medios de comunicación, que se han vuelto muy poderosos.

c. El autor considera que, si bien se puede creer que la revolución de la burguesía ha sido derrotar al comunismo, existe una revolución más fuerte aún que consiste en controlar los medios de comunicación.

\section{Pregunta 3}

En el párrafo 2, Feinmann plantea una serie de preguntas: “¿Hay una verdad en este mundo? ¿Existe la verdad? ¿Hay una verdad única? ¿Hay una verdad para todos?"

¿Por qué el autor plantea estas preguntas?

Seleccione una respuesta.

a. El autor plantea estas preguntas para expresar interrogantes que no tienen respuestas.

b. El autor plantea estas preguntas con el objetivo principal de llamar la atención del lector.

c. El autor plantea estas preguntas para introducir una cuestión polémica, es decir, una cuestión que actualmente es conflictiva en la sociedad y a partir de la cual se generan diferentes posturas y opiniones.

\section{Pregunta 4}

La HIPÓTESIS principal de este texto es: 
Seleccione una respuesta.

a. Los medios tienen poder.

b. Estamos viviendo una revolución comunicacional, que consiste en que los medios de comunicación, que responden a los intereses de la burguesía capitalista, imponen sus ideas en la sociedad.

c. Las empresas mediáticas se construyen con determinados capitales y con determinada ideología.

\section{Pregunta 5}

Inicialmente, el autor reflexiona sobre la idea de "verdad" articulando un ARGUMENTO que se podría sintetizar del siguiente modo:

No existe "la verdad" sobre un hecho, sino tantas verdades como grupos que estén interesados en hablar de ese hecho. Sin embargo, lo que suele suceder es que uno de los grupos logra imponer su visión de los hechos por sobre los demás y así hacen creer que su verdad es la única. En este sentido, la burguesía, a través del dominio de los medios de comunicación, impone actualmente su verdad.

Indicar cuál de las siguientes citas sería más adecuada para reemplazar la última oración del argumento (es decir, para colocar en lugar de esa oración):

Seleccione una respuesta.

a. En este sentido, Feinmann expresa lo siguiente: "La existencia es una lucha de verdades. Hay una frase de Nietzsche, del cual soy adherente, que dice: 'No hay hechos, hay interpretaciones'".

b. En este sentido, Feinmann explica lo siguiente: "El poder es conquistar, es conseguir que mi verdad sea la verdad de la mayoría, y si es posible, de todos. Esta es una lucha que todos los medios de comunicación están empeñados en ganar, y es el gran triunfo de la burguesía mediática del fin del milenio y sobre todo de esta primera década del siglo XXI".

c. En este sentido, Feinmann indica lo siguiente: "O sea: de cada hecho habrá tantas interpretaciones como grupos interesados en interpretarlo existan. Si yo consigo dominar o tener la mayoría de los medios de comunicación, de cada hecho, de cada suceso, voy a imponer mi opinión por sobre todos los demás".

\section{Pregunta 6}

En el párrafo 10 del texto, se hace referencia a un periodista al que el director manda a hacer una nota sobre una obra de teatro y debe modificarla porque su crítica atenta contra los intereses de la empresa.

Indicar qué ARGUMENTO ilustra este ejemplo:

Seleccione una respuesta.

a. Actualmente los periodistas no son independientes ideológicamente. Las grandes empresas periodísticas controlan lo que dicen y escriben sus empleados, incluidos los periodistas. 
b. La verdad es una creación del poder, de quienes imponen su verdad como absoluta.

c. Los medios imponen su verdad así como la Iglesia Católica imponía la verdad de Dios en el Medioevo.

\section{Anexo 2. Cuestionario 2}

\section{Pregunta 1}

La cuestión que desencadena la argumentación está enunciada en:

Seleccione una respuesta.

a. El primer párrafo.

b. El segundo párrafo.

c. El título.

\section{Pregunta 2}

La hipótesis principal del texto es:

Seleccione una respuesta.

a. El agua es de todos.

b. El agua es de las grandes empresas que administran ese recurso natural.

c. El agua es de todos pero, en los hechos, algunas grandes empresas se apropian de este recurso con la ayuda de la ONU.

\section{Pregunta 3}

En el párrafo 2 del texto se hace referencia al lanzamiento de la iniciativa "Water Mandate" por parte de la ONU. ¿Qué argumento es ilustrado por este ejemplo?

Seleccione una respuesta.

a. Marck \& Co. o Siemens son empresas que privatizan bienes que son de todos. b. El propósito de esta resolución es loable porque garantiza el cuidado de un recurso no renovable.

c. Los megapolios se amparan en resoluciones de Naciones Unidas para obtener beneficios económicos de bienes que son de todos.

\section{Pregunta 4}

En el párrafo 3 del texto se hace referencia a que el Banco Mundial asumió el control del Fondo Verde de la Conferencia sobre el Cambio Climático, financiado con 100 mil millones de dólares. Indicar qué argumento es ilustrado por este ejemplo.

Seleccione una respuesta.

a. Los países más poderosos y las grandes empresas privadas asociadas a estos influyen en la ONU porque financian sus actividades, ya que este organismo carece de fondos propios.

b. El Banco Mundial no se ha caracterizado por la transparencia en el manejo de fondos públicos.

c. Muchos fondos asignados a proyectos importantes de la ONU son administrados por el FMI, el Banco Mundial y bancos de desarrollo regionales, a pesar de su poca transparencia.

\section{Pregunta 5}

En el texto, el autor incluye palabras de la ecologista canadiense Maud Barlow. ¿Cuál es la función principal que cumple esta cita? 
Seleccione una respuesta.

a. Advertir que se aproxima una crisis relacionada con el agua.

b. Incluir la opinión de una especialista en el tema para sostener su hipótesis.

c. Presentar otros ejemplos que ilustren sus argumentos.

\section{Pregunta 6}

En el último párrafo, el autor refiere el caso de la Alcaldía de París, que en junio volvió a adquirir los servicios de agua administrados en forma privada por las compañías Veolia y Suez. Luego, concluye: "Pero no siempre la rapacidad encuentra freno". ¿Qué significa esta conclusión, en relación a este caso y a los ejemplos presentados anteriormente en el texto?

Seleccione una respuesta.

a. Que la actitud de la Alcaldía de París constituye otro caso de rapacidad sin límites.

b. Que la decisión de la Alcaldía de París constituye un ejemplo de cómo se puede y se debe limitar la rapacidad del sector privado.

c. Que la decisión de la Alcaldía de París constituye un ejemplo de cómo no siempre se puede limitar la rapacidad del sector privado. 